\title{
Light vector mesons in PHENIX
}

\section{Yoshihide Nakamiya* for the PHENIX Collaboration}

Hiroshima University, 1-3-1 Kagamiyama, Higashi-hiroshima, Hiroshima, Japan

E-mail: nakamiya@hepl.hiroshima-u.ac.jp

The production of light vector mesons is important to research the property of the phase transition between normal nuclear matter and deconfined partonic matter because mass modification is expected to happen for these mesons in high energy density medium. We present recent results of $\phi$ and $\omega$ production via various decay channels in $\mathrm{p}+\mathrm{p}, \mathrm{d}+\mathrm{Au}$ and $\mathrm{Au}+\mathrm{Au}$ collisions at $\sqrt{s_{N N}}$ $=200 \mathrm{GeV}$ at the RHIC-PHENIX experiment.

Critical Point and Onset of Deconfinement - 4th International Workshop

July 9 - 13, 2007

Darmstadt, Germany

${ }^{*}$ Speaker. 


\section{Introduction}

High energy heavy-ion collision is an attractive tool to investigate dynamics of the strongly interacting matter governed by the partonic degree of freedom at high energy density. Recent results [1] from experiments at RHIC suggest the existence of the strongly interacting partonic matter at a very high energy density. Among the many diagnostic probes, the production of light vector mesons still plays an important rule. Mass modification (mass, lifetime and branching ratio) of light vector mesons is expected to happen under the extreme condition. Measurement of light vector mesons is also important from the viewpoint that RHIC full energy collision covers the crossover region in the QCD phase diagram [2]. Especially, electromagnetic probes such as leptons and photons are clean keys to research the properties of the created partonic matter, as they penetrate in the medium with less interaction due to their electromagnetic coupling. Naturally measurement of light vector mesons via leptonic decay provides us with intrinsic informations for the properties of medium. And also such properties become clearer by compared to results for other decay channels.

\section{Experimental Setup}

The PHENIX detector is designed to perform a broad study of proton+proton to $\mathrm{Au}+\mathrm{Au}$ collisions with a broad range of energy from $\sqrt{s_{N N}}=22.5 \mathrm{GeV}$ to $500 \mathrm{GeV}(200 \mathrm{GeV}$ is maximum energy for ion). The PHENIX detector comprises a global detector, two central spectrometers and two muon spectrometers. A global detector consists of Zero-Degree Calorimeters (ZDC) and Beam-Beam Counters (BBC). BBC provides the minimum bias event trigger and determines the $\mathrm{z}$-coordinate of the collision vertex. Event centrality is determined by the combination of BBC and ZDC. The central spectrometers, which cover the pseudo-rapidity range of $|\eta|<0.35$ and 90 degrees in azimuthal angle, have a capability to measure electrons, photons as well as hadrons at the same time. Drift Chamber (DC) and the first layer of Pad Chamber (PC1) is used for reconstruction of charged particle momentum. Time-Of-Flight (TOF) subsystems covering half of the east arm and Electro Magnetic Calorimeter (EMC) covering both arms are used for hadron identification. The TOF subsystems and time of flight by EMC identify kaon within $0.3<p_{T}<2.0$ $\mathrm{GeV} / \mathrm{c}$ and $0.3<p_{T}<1.0 \mathrm{GeV} / \mathrm{c}$ respectively. Electron is separated from pion by Ring-Imaging CHerenkov ( $\mathrm{RICH})$ counter in the momentum rage of $0<p_{T}<4.7 \mathrm{GeV} / \mathrm{c}$. Matching between measured energy deposit and momentum is also used for electron identification.

\section{Analysis}

The di-electron analysis mainly consists of electron identification and reconstruction of invariant mass. The invariant mass spectrum is calculated from combinations of electrons and positrons. Naturally it includes the signal and the combinatorial background. The difficulty of electron analysis is that multiplicity is enormous especially in case of $\mathrm{Au}+\mathrm{Au}$ collisions and electrons are generated anywhere. The most part of generated electrons originates from Dalitz decay of $\pi^{0}$ mesons and $\eta$ mesons and from photon conversion at the beam pipe. As a result, combinatorial backgrounds become huge. In addition, ghost tracks which arise in RICH make an undesirable correlated peak in the invariant mass spectrum. It affects normalization between the foreground and the background 
in the low mass region. Thus this analysis focuses on rejecting these backgrounds. Details are discussed in ref[3]. After these improvements, raw yield of $\omega(\phi) \rightarrow e^{+} e^{-}$is counted around known particle masses after subtracting mixed event distribution from invariant mass spectra.

For $\phi \rightarrow K^{-} K^{+}$analysis, kaon identification is performed by measuring the time of flight of charged particles. Invariant mass spepctra are reconstructed after kaon identification and background shape is estimated by event mixing technique. Raw yield of $\phi \rightarrow K^{-} K^{+}$is counted by known particle masses.

For $\omega \rightarrow \pi^{0} \gamma$ and $\omega \rightarrow \pi^{0} \pi^{+} \pi^{-}$analysis, At first, we selected $\pi^{0}$ candidates reconstructing of the invariant mass by two $\gamma$. It is performed by combining EMC clusters produced photon originated from the collision vertex. These $\pi^{0}$ candidates are combined with the third photon or identified charged pion and they reconstructed the invariant mass of $\omega$ mesons. After that, Raw yield of $\omega \rightarrow \pi^{0} \gamma$ and $\omega \rightarrow \pi^{0} \pi^{+} \pi^{-}$is counted by known particle masses.

\section{Result}

\subsection{Invariant $m_{T}\left(p_{T}\right)$ spectra}

The invariant $m_{T}\left(p_{T}\right)$ spectra of light vector mesons via various dacay channels at various collision systems has been measured in the PHENX experiment. In Fig. 1, the top-left plot shows $m_{T}$ spectra of $\phi \rightarrow e^{+} e^{-}$decay channels for each centrality at $\mathrm{Au}+\mathrm{Au} 200 \mathrm{GeV}$ and the top-right one [4] is $\phi \rightarrow K^{+} K^{-}$for each centrality at $\mathrm{Au}+\mathrm{Au} 200 \mathrm{GeV}$, respectively. The bottom-left plot indicates $m_{T}$ spectra of $\phi \rightarrow e^{+} e^{-}$at $\mathrm{d}+\mathrm{Au} 200 \mathrm{GeV}$ and the bottom-right one is $\phi \rightarrow K^{+} K^{-}$at $\mathrm{d}+\mathrm{Au} 200 \mathrm{GeV}$, respectively. In Fig. 2, the top-left plot shows $p_{T}$ spectra of $\omega \rightarrow e^{+} e^{-}$decay channels at minimum bias in $\mathrm{Au}+\mathrm{Au} 200 \mathrm{GeV}$ and the top-right one is $\omega \rightarrow \pi^{0} \gamma$ for each centrality at $\mathrm{Au}+\mathrm{Au} 200 \mathrm{GeV}$, respectively. the bottom-left plot indicates $p_{T}$ spectra of $\omega \rightarrow \pi^{0} \gamma$ and $\omega \rightarrow$ $\pi^{0} \pi^{+} \pi^{-}$in d+Au and p+p 200GeV. The bottom-right one shows $\omega \rightarrow \pi^{0} \gamma$ and $\omega \rightarrow \pi^{0} \pi^{+} \pi^{-}$in $\mathrm{p}+\mathrm{p} 200 \mathrm{GeV}$.

\subsection{Results of line shape analysis}

In this section, we present results of line shape analysis for $\phi$ mesons and $\omega$ mesons [4, 5]. Fig. 3 shows measured mass centroid and width for $\phi \rightarrow K^{+} K^{-}$as a function of number of participant nucleons in $\mathrm{Au}+\mathrm{Au}$ collisions at $\sqrt{s_{N N}}=200 \mathrm{GeV}$. As a result, there is no centrality dependence of $\phi$ mass centroid within less than $1 \mathrm{MeV} / c^{2}$. For mass width, there is no convincing evidence of a variation of the $\phi$ width as a function of number of participant nucleons. Fig. 4 shows measured mass centroid and width for $\omega \rightarrow \pi^{0} \pi^{+} \pi^{-}$as a function of $p_{T}$ in $\mathrm{d}+\mathrm{Au}$ collisions (upper panel) and $\mathrm{p}+\mathrm{p}$ collisions (lower panel) at $200 \mathrm{GeV}$. In both cases, there is no mass modification as a function of $p_{T}$ and agree with the PDG value.

\subsection{The invariant yield}

The left plot in Fig. 5 shows the centrality dependence of the invariant $\phi$ yield per participant pair for $\phi \rightarrow e^{+} e^{-}$decay channels and $\phi \rightarrow K^{+} K^{-}$decay channels in $\mathrm{Au}+\mathrm{Au}$ and $\mathrm{d}+\mathrm{Au}$ collisions at $\sqrt{s_{N N}}=200 \mathrm{GeV}$. The $\phi$ yield are extracted from the invariant $m_{T}$ distribution. The comparison of the $\phi$ meson production measured via $e^{+} e^{-}$and $K^{+} K^{-}$decay channels may show 
a possible increase of the yield in the di-electron channel compared with kaon one. However we cannot make definite statement with regard to difference between the yield of two decay channels due to large errors. The invariant yields obtained by both decay channels are consistent and have no centrality dependence within error bars. In addition, the $\phi$ yield of $\mathrm{Au}+\mathrm{Au}$ peripheral collisions is almost same compared with that of $\mathrm{d}+\mathrm{Au}$ collisions as a reference data. The right plot in Fig. 5 represents temperature of $\phi$ mesons as a function of the number of participant nucleons. Temperature is obtained by the invariant $m_{T}$ spectra. The temperature measured in Au+Au collisions through $e^{+} e^{-}$and $K^{+} K^{-}$decay channels is centrality independent and agree within the statistical and systematical uncertainties.

Fig. 6 shows the centrality dependence of the invariant $\omega$ yield per participant pair for $e^{+} e^{-}$ decay channels in $\mathrm{Au}+\mathrm{Au}$ collisions at $\sqrt{s_{N N}}=200 \mathrm{GeV}$. The invariant yield of $\omega$ mesons is found to scale with the number of participant nucleons. 

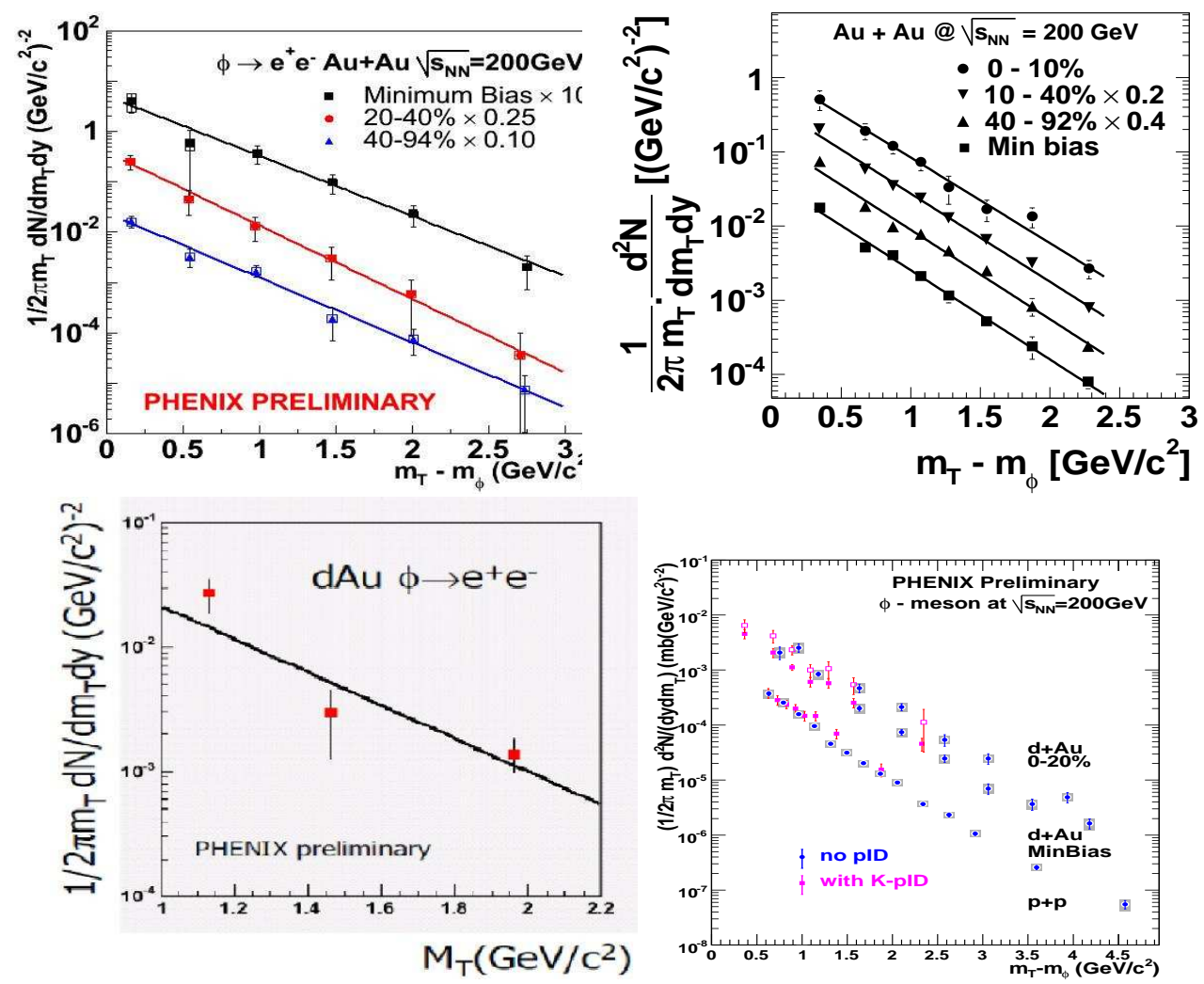

Figure 1: Invariant $m_{T}$ spectra for $\phi$ mesons measured in $\mathrm{p}+\mathrm{p}, \mathrm{d}+\mathrm{Au}$ and $\mathrm{Au}+\mathrm{Au}$ collisions at $\sqrt{s_{N N}}=200$ $\mathrm{GeV}$.
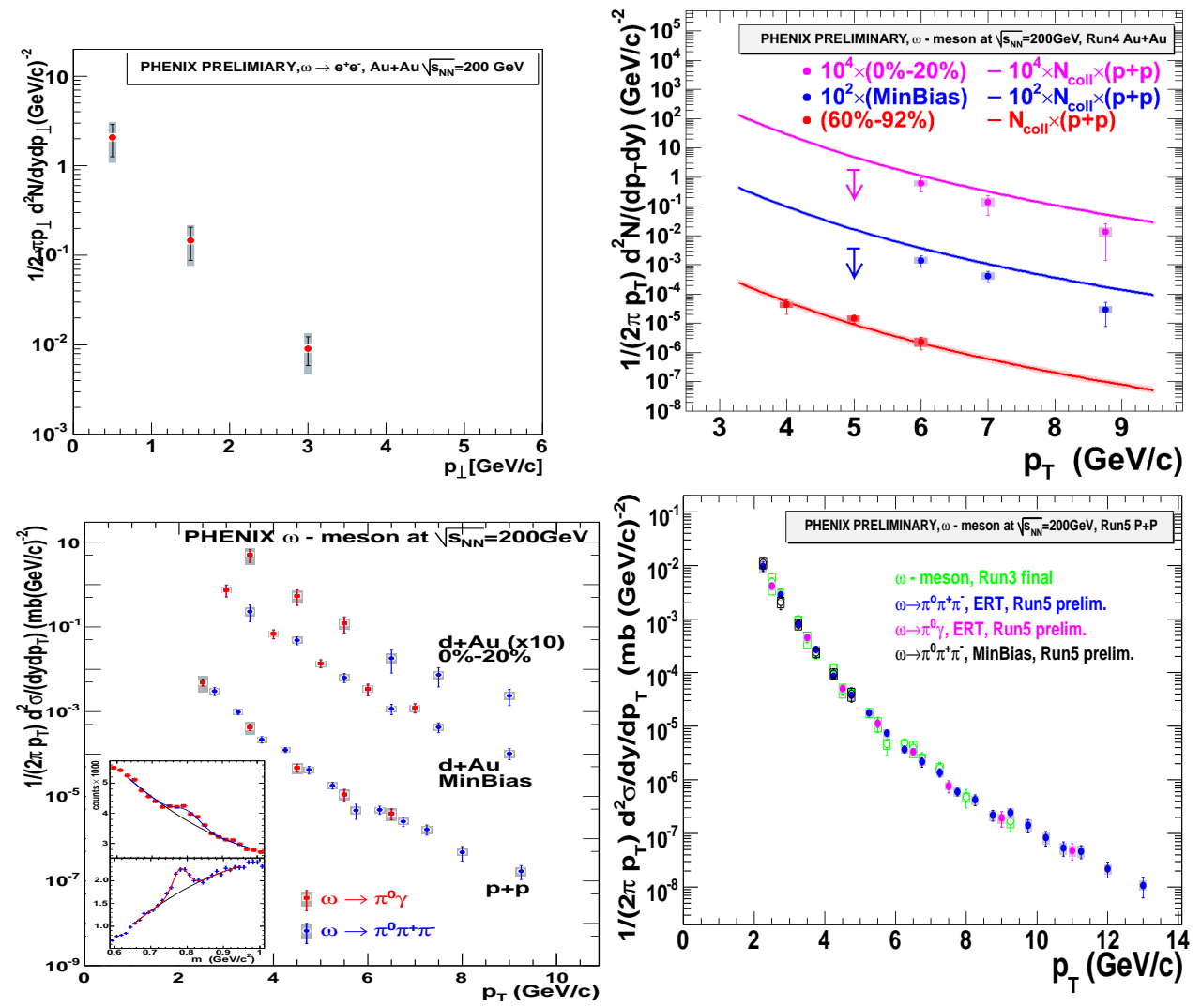

Figure 2: Invariant $p_{T}$ spectra for $\omega$ mesons measured in $\mathrm{p}+\mathrm{p}, \mathrm{d}+\mathrm{Au}$ and $\mathrm{Au}+\mathrm{Au}$ collisions at $\sqrt{s_{N N}}=200$ $\mathrm{GeV}$. 

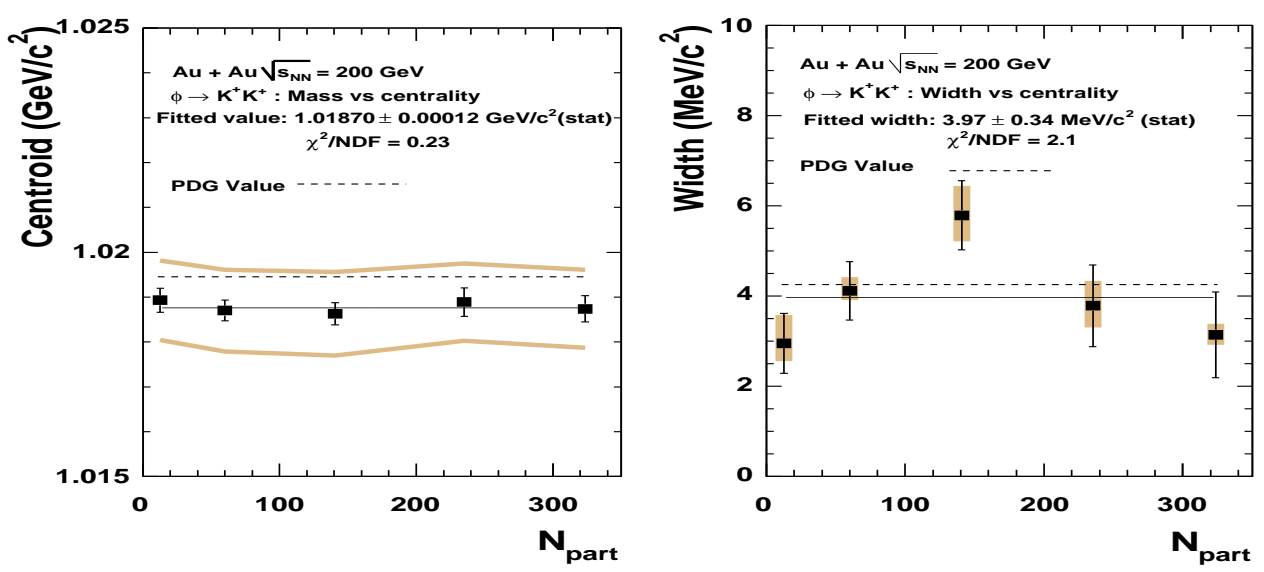

Figure 3: $\phi$ mass centroid (left panel) and width (right panel) as a function of number of participant nucleons. For the mass centroid plot, the $1 \sigma$ systematic errors limits on the data points are shown by two continuous bands. The dotted line shows the PDG value and the solid line indicates the centroid value obtained from a one-parameter-fit assumption. For the width plot, the systematic errors on the Relativistic Breit-Wigner width are indicated as bands on each data point. Dotted line is the PDG width and the solid line shows a one-parameter-fit result for the measured data points.

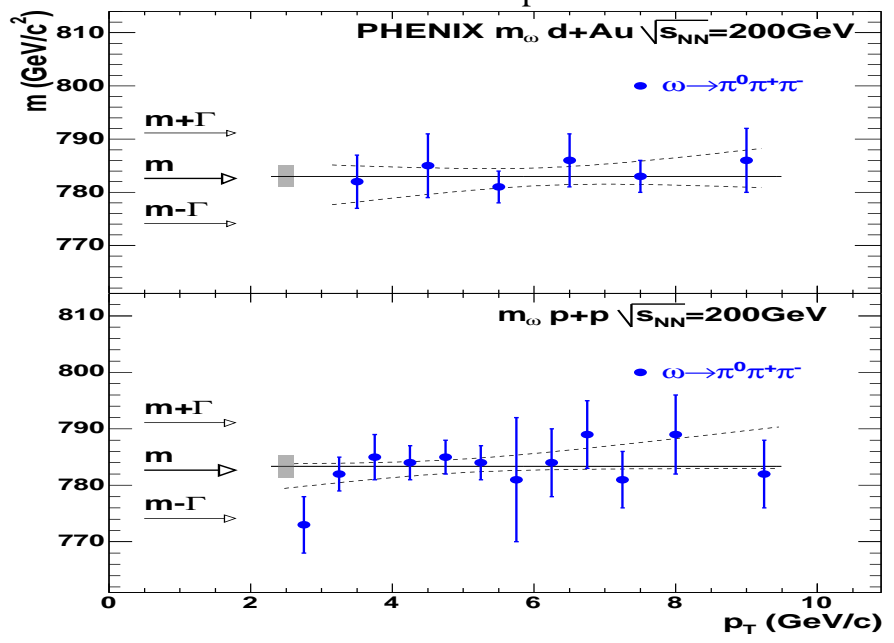

Figure 4: Reconstructed $\omega$ mass measured in the $\pi^{0} \pi^{+} \pi^{-}$decay channel as a function of $p_{T}$ in $d+A u$ (upper panel) and p+p (lower panel) collisions at $\sqrt{s_{N N}}=200 \mathrm{GeV}$. Error bars show statistical errors. Straight line is the result of fitting with data point to a constant. Dashed line shows values within $1 \sigma$ of the best linear fit to the data. Boxes on the left shows systematic error on the fit values. PDG values for $\omega$ mesons mass(m) and width $(\Gamma)$ are shown with arrows on the left [6]. 

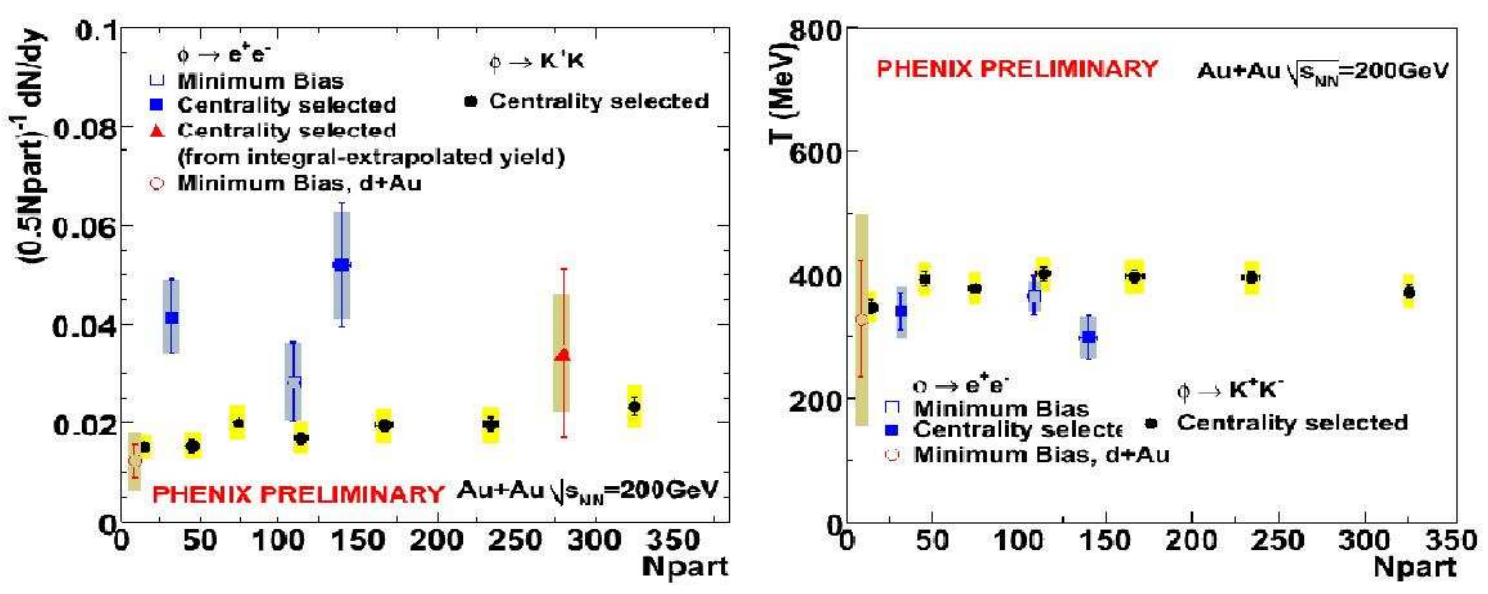

Figure 5: The left figure is the invariant $\phi$ yield per unit rapidity as a function of number of participant nucleons in $\mathrm{Au}+\mathrm{Au}$ and $\mathrm{d}+\mathrm{Au}$ collisions at $200 \mathrm{GeV}$ for $\phi \rightarrow e^{+} e^{-}$and $\phi \rightarrow e^{+} e^{-}$. Data points are scaled by $0.5 \times$ the number of participant nucleons within errors. Statistical errors are shown with error bar and systematic errors are shown with error band. The right figure is temperature for $\phi$ mesons as a function of number of participant nucleons.

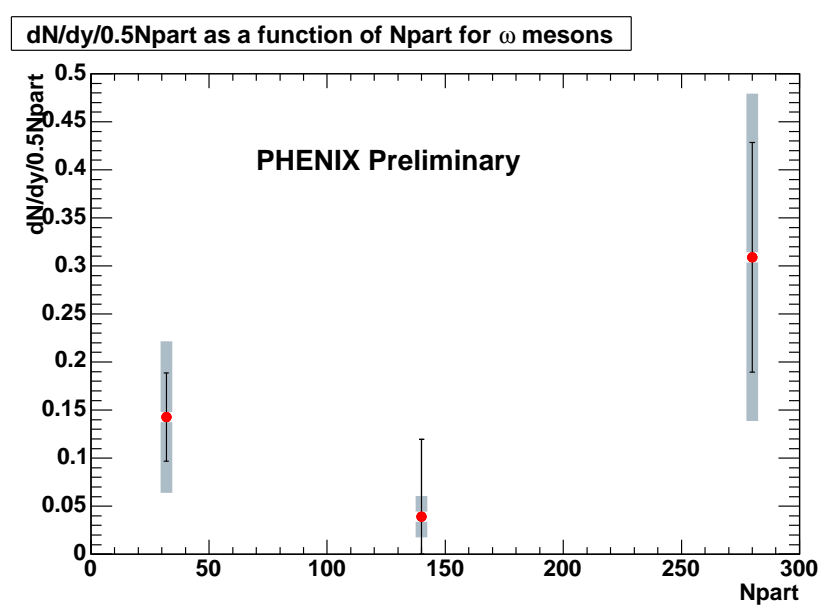

Figure 6: The invariant $\omega$ yield per unit rapidity as a function of number of participant nucleons in $\mathrm{Au}+\mathrm{Au}$ collisions at $200 \mathrm{GeV}$. Data points are scaled by $0.5 \times$ the number of participant nucleons. Statistical errors are shown with error bar and systematic errors are shown with error band. 


\section{Summary}

In summary we have presented measurement of $\phi$ mesons and $\omega$ mesons via various decay channels $\left(\phi \rightarrow e^{+} e^{-}, \phi \rightarrow K^{+} K^{-}, \omega \rightarrow e^{+} e^{-}, \omega \rightarrow \pi^{0} \gamma\right.$ and $\left.\phi \rightarrow \pi^{0} \pi^{+} \pi^{-}\right)$in $\mathrm{p}+\mathrm{p}, \mathrm{d}+\mathrm{Au}$ and $\mathrm{Au}+\mathrm{Au}$ collisions at $\sqrt{s_{N N}}=200 \mathrm{GeV}$. As a result there is no convincing mass modification for line shape of $\phi \rightarrow K_{+} K_{-}$in $\mathrm{Au}+\mathrm{Au} 200 \mathrm{GeV}$ and $\omega \rightarrow \pi^{0} \pi^{+} \pi^{-}$in p+p and d+Au 200GeV. Results agree with the PDG value within errors. The invariant yield of $\phi$ mesons and $\omega$ mesons in $\mathrm{Au}+\mathrm{Au}$ $200 \mathrm{GeV}$ is independent on number of participants nucleons within errors.

\section{References}

[1] K. Adcox et al. (PHENIX Collaboration), Nuclear Physics A Volume 757, Issues 1-2, 8 August 2005 , Pages 184-283, 2005-05-24

[2] Y.Aoki, Z.Fodor et al. Phys. Lett. B 643 (2006) 46-54

[3] A.Toia et al. (PHENIX Collaboration), Proceedings of Critical Point and Onset of Deconfinement 2007

[4] S.S. Adler et al. (PHENIX Collaboration), Phys. Rev. C 72014903 (2005)

[5] S.S. Adler et al. (PHENIX Collaboration), Phys. Rev. C 75051902 (2007)

[6] Particle Data Group. S. Edelman. et al. Phys. Lett. B592, 1 (2004) 